\title{
BMJ Open Effects of multiparity on left ventricular diastolic dysfunction in women: cross- sectional study of the KoRean wOmen'S chest pain rEgistry (KoROSE)
}

\author{
Hyun-Jin Kim, ${ }^{1}$ Myung-A Kim, ${ }^{2}$ Hack-Lyoung Kim, ${ }^{2}$ Wan Joo Shim, ${ }^{3}$ \\ Seong Mi Park, ${ }^{3}$ Mina Kim, ${ }^{3}$ Hyun Ju Yoon, ${ }^{4}$ Mi Seung Shin, ${ }^{5}$ Kyung-Soon Hong, ${ }^{6}$ \\ Gil Ja Shin, ${ }^{7}$ Yong-Hyun Kim, ${ }^{8}$ Jin Oh $\mathrm{Na},{ }^{8}$ Jin-Ok Jeong ${ }^{9}$
}

To cite: Kim H-J, Kim M-A Kim H-L, et al. Effects of multiparity on left ventricular diastolic dysfunction in women: cross-sectional study of the KoRean w0men'S chest pain rEgistry (KoROSE). BMJ Open 2018;8:e026968. doi:10.1136/ bmjopen-2018-026968

- Prepublication history and additional material for this paper are available online. To view these files, please visit the journal online (http://dx.doi. org/10.1136/bmjopen-2018026968).

Received 28 September 2018 Revised 11 November 2018 Accepted 21 November 2018

Check for updates

(C) Author(s) (or their employer(s)) 2018. Re-use permitted under CC BY-NC. No commercial re-use. See rights and permissions. Published by BMJ.

For numbered affiliations see end of article.

Correspondence to

Dr Myung-A Kim;

kma@snu.ac.kr

\section{ABSTRACT}

Objectives To investigate the association between left ventricular (LV) diastolic dysfunction and multiparity in patients with suspected coronary artery disease (CAD). Design Cross-sectional study.

Setting Linked secondary and tertiary care records from 29 cardiac centres which participated in KoRean w0men's chest pain rEgistry.

Participants 960 women with suspected CAD who underwent invasive coronary angiography from February 2011 to May 2017. The patients were classified by parity number, as follows: low-parity, 0 to $<3$; multiparity, $\geq 3$ pregnancies.

Main outcome measure Prevalence of LV diastolic dysfunction.

Results There were 302 and 658 low-parity and multiparity patients, respectively. The prevalence of LV diastolic dysfunction was significantly higher in the multiparity than in the low-parity group. The multiparity group had significantly lower $E$ and e' septal velocities and E/A ratio, and had a significantly higher E/e' ratio and right ventricular systolic pressure, which are parameters of LV diastolic dysfunction, than the low-parity group. The prevalence of CAD was significantly higher in the multiparity than in the low-parity group. Receiver operating characteristic curve analysis identified a parity of 2.5 as the cut-off for predicting LV diastolic dysfunction (area under the curve, 0.66; sensitivity, 74.1\%; specificity, $52.0 \%$; $95 \%$ Cl 0.607 to 0.706 ; $p<0.001$ ). After adjustment for confounding factors, multivariate regression analysis showed that multiparity had a 1.80-fold increased risk for LV diastolic dysfunction (OR 1.80, 95\% Cl 1.053 to $3.081, p=0.032$ ).

Conclusions The prevalence of LV diastolic dysfunction was higher in multiparity than in low-parity women with suspected CAD. Multiparity was an independent risk factor for LV diastolic dysfunction. LV diastolic dysfunction should be evaluated in multiparous women for the risk of subsequent cardiovascular disease and facilitate the initiation of appropriate treatment.

\section{INTRODUCTION}

Left ventricular (LV) diastolic dysfunction has been known to play an important role
Strengths and limitations of this study

- The strength of our study is definitively confirmed that haemodynamic and physiological changes in patients with multiparity are associated with longterm diastolic dysfunction.

- This study has a strength of 1:1 age-matched analysis of low-parity and multiparity groups to compensate for the effect of age on diastolic dysfunction.

- This has been a cross-sectional study which inherently cannot clarify the causal relationships.

- This study has been performed in patients suspected of having coronary artery disease undergoing coronary angiography.

in development of heart failure and is also related to cardiovascular mortality. ${ }^{1}{ }^{2} \mathrm{LV}$ diastolic dysfunction is characterised by abnormal LV relaxation, reduced LV distensibility or increased LV filling. ${ }^{3}$ Doppler echocardiography is appropriately and widely used for assessment of parameters in patients presenting with LV diastolic function.

Several haemodynamic changes are associated with pregnancy, including increased maternal cardiac output, increased preload and circulatory blood volume, and decreased systemic vascular resistance. ${ }^{4}$ In accordance with these changes, LV wall thickness and LV mass increase during pregnancy, resulting in LV hypertrophy. ${ }^{5}$ These changes represent cardiovascular adaptation for maintaining the pregnancy. However, these loading conditions have been found to cause LV diastolic dysfunction during pregnancy. ${ }^{5}{ }^{6}$ Although the complete recovery of this deterioration after delivery is known to occur, previous studies have shown that repeated pregnancies are a risk factor for cardiovascular disease in women. ${ }^{78}$ In addition, pregnancy induces hormonal changes that include 
changes in the levels of insulin and gestational hormones. The hormonal effects can lead to changes in the blood levels of lipids and glucose and changes in body weight, increasing the risk of the development of coronary artery disease. ${ }^{9-11}$ As an early change in all cardiovascular diseases, LV diastolic dysfunction has also been known to be associated with coronary artery disease. We hypothesised that the haemodynamic and physiological changes during repeated pregnancies and repeated cardiovascular adaptation to volume overload might affect longterm LV diastolic function. Few studies have definitively confirmed that multiparity is involved with long-term LV diastolic dysfunction. Therefore, we investigated the association between multiparity and LV diastolic dysfunction in women with suspected coronary artery disease.

\section{METHODS}

\section{Study population}

Cross-sectional study data were obtained from the database of the KoRean wOmen'S chest pain rEgistry (KoROSE). We accessed the database of the KoROSE using electronic case report form (CRF) which is an electronic version of the traditional CRF. From February 2011 to May 2017, 29 cardiac centres in Korea participated in this registry. The investigators of each cardiac centre which were approved by the institutional review board (IRB) could type directly into fields using a computer and transmitted electronically using electronic CRF. Patients who visited with chest pain were aged 20 years or older, and underwent invasive coronary angiography for detecting coronary artery disease were enrolled in the registry. ${ }^{12}$ Patients who had end-stage renal disease, malignancy, primary pulmonary hypertension, chronic obstructive pulmonary disease or autoimmune disease were excluded from the database. Among a total of 2311 patients (men=681, women=1630), 960 women with information about their parity were finally analysed in this study. We classified the patients into groups according to parity number, as follows: low-parity group (parity 0 to $<3$ ) and multiparity group (parity $\geq 3$ ). We also extracted 1:1 age-matched low-parity and multiparity groups from this population. The study protocol complied with the Declaration of Helsinki.

\section{Clinical and laboratory assessments}

Patients' demographic and clinical characteristics were obtained from the data. The following demographic and clinical characteristics were extracted: age, parity, body mass index $\left(\mathrm{BMI} ; \mathrm{kg} / \mathrm{m}^{2}\right)$, waist circumference, systolic blood pressure, diastolic blood pressure, heart

Table 1 Baseline characteristics

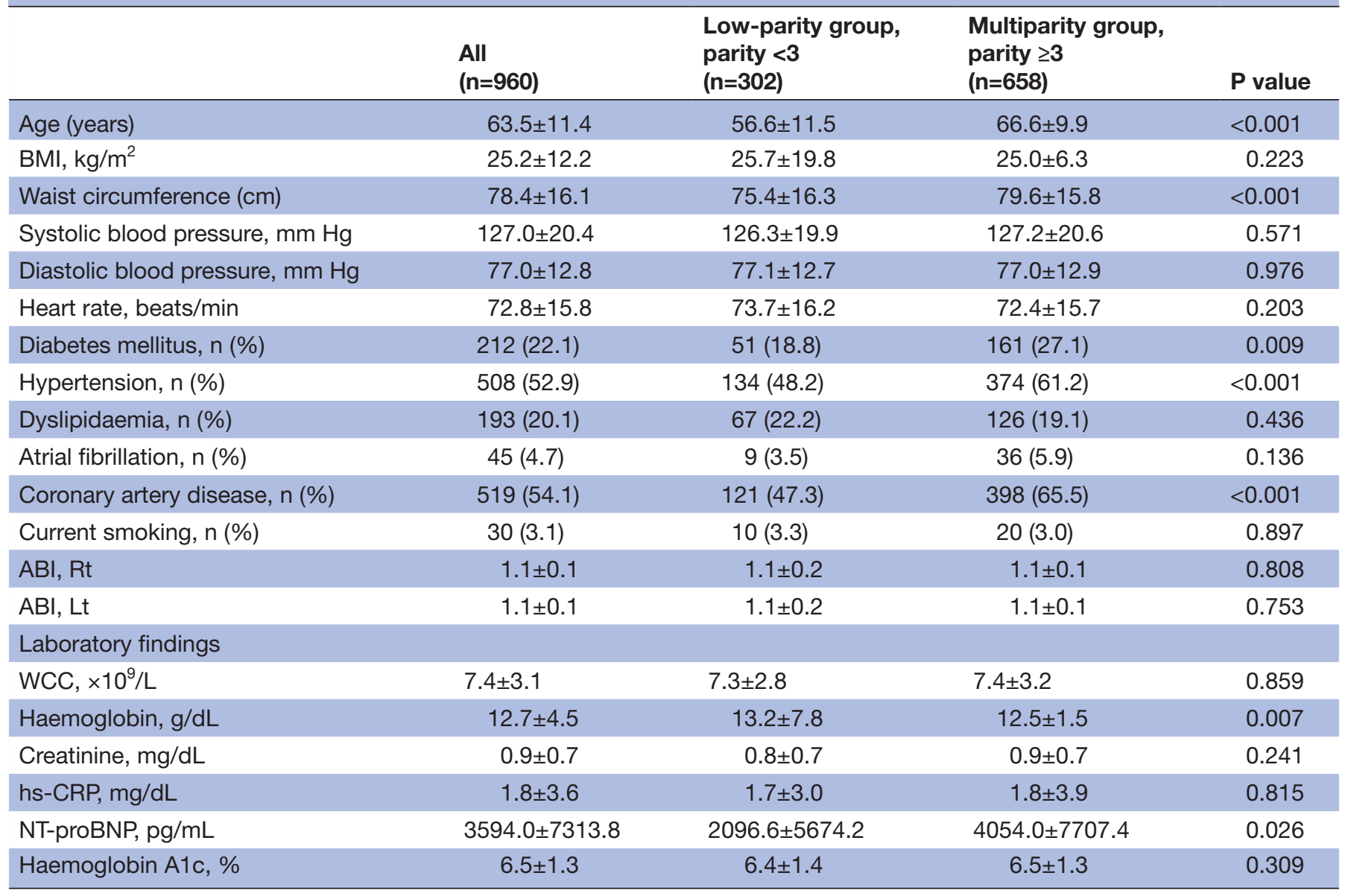

$\mathrm{ABI}$, ankle-brachial index; BMI, body mass index; hs-CRP, high sensitivity-C-reactive protein; Lt, left; NT-proBNP, NT- pro-brain-type natriuretic peptide; Rt, right; WCC, white cell count. 
Table 2 Echocardiographic measurements

\begin{tabular}{|c|c|c|c|c|}
\hline & $\begin{array}{l}\text { All } \\
(n=960)\end{array}$ & $\begin{array}{l}\text { Low-parity group, } \\
\text { parity <3 } \\
(\mathrm{n}=302)\end{array}$ & $\begin{array}{l}\text { Multiparity group, } \\
\text { parity } \geq 3 \\
(\mathrm{n}=658)\end{array}$ & $P$ value \\
\hline Left ventricular end-diastolic dimension, $\mathrm{mm}$ & $47.3 \pm 5.0$ & $46.3 \pm 4.0$ & $47.7 \pm 5.4$ & $<0.001$ \\
\hline Left ventricular end-systolic dimension, mm & $30.2 \pm 6.4$ & $29.1 \pm 5.2$ & $30.7 \pm 6.8$ & 0.002 \\
\hline Interventricular septum thickness, mm & $9.3 \pm 1.6$ & $9.1 \pm 1.5$ & $9.3 \pm 1.7$ & 0.008 \\
\hline Posterior wall thickness, $\mathrm{mm}$ & $9.3 \pm 4.3$ & $9.1 \pm 4.8$ & $9.4 \pm 4.0$ & $<0.001$ \\
\hline Left ventricular ejection fraction, $\%$ & $59.3 \pm 9.8$ & $61.0 \pm 9.0$ & $58.6 \pm 10.0$ & 0.004 \\
\hline Left ventricular mass index, $\mathrm{g} / \mathrm{m}^{2}$ & $101.8 \pm 34.8$ & $95.8 \pm 29.3$ & $105.0 \pm 37.1$ & 0.001 \\
\hline Left atrial dimension, $\mathrm{mm}$ & $37.6 \pm 6.3$ & $35.9 \pm 5.9$ & $38.4 \pm 6.3$ & $<0.001$ \\
\hline $\mathrm{E}, \mathrm{cm} / \mathrm{s}$ & $66.7 \pm 20.1$ & $69.1 \pm 20.1$ & $65.7 \pm 20.0$ & 0.019 \\
\hline $\mathrm{A}, \mathrm{cm} / \mathrm{s}$ & $77.5 \pm 22.8$ & $71.7 \pm 19.1$ & $80.2 \pm 23.9$ & $<0.001$ \\
\hline E/A ratio & $1.0 \pm 0.6$ & $1.0 \pm 0.4$ & $0.9 \pm 0.7$ & $<0.001$ \\
\hline Deceleration time, $\mathrm{ms}$ & $212.1 \pm 56.1$ & $206.7 \pm 60.5$ & $214.5 \pm 53.9$ & 0.030 \\
\hline$e^{\prime}$ septal, $\mathrm{cm} / \mathrm{s}$ & $5.9 \pm 2.1$ & $6.7 \pm 2.4$ & $5.5 \pm 1.9$ & $<0.001$ \\
\hline E/e' ratio & $12.6 \pm 6.1$ & $11.4 \pm 5.3$ & $13.1 \pm 6.4$ & $<0.001$ \\
\hline RVSP, mm Hg & $32.1 \pm 9.6$ & $29.9 \pm 8.6$ & $33.0 \pm 9.9$ & $<0.001$ \\
\hline TR peak velocity, $\mathrm{cm} / \mathrm{s}$ & $2.3 \pm 0.3$ & $2.2 \pm 0.3$ & $2.3 \pm 0.3$ & 0.038 \\
\hline Grade of left ventricular diastolic dysfunction, n (\%) & & & & $<0.001$ \\
\hline Normal & $148(15.4)$ & $77(33.0)$ & $71(13.7)$ & \\
\hline 1 & $518(54.0)$ & $129(55.4)$ & $389(75.2)$ & \\
\hline $2-3$ & $84(8.8)$ & 27 (11.6) & $57(11.1)$ & \\
\hline
\end{tabular}

RVSP, right ventricular systolic pressure; TR, tricuspid regurgitation.

rate, ankle-brachial index (ABI) and current smoking status; as well as traditional cardiovascular risk factors which included history of diabetes mellitus, hypertension, dyslipidaemia, atrial fibrillation and coronary artery disease. The traditional angiographic imaging definition was used for coronary artery disease which defined focal coronary artery stenosis of $50 \%$ or more vessel diameter. ${ }^{13}$ Each patient's BMI was calculated from her height and weight. The following laboratory data were extracted: white blood cell count, and haemoglobin, creatinine, high sensitivity-C-reactive protein, NT-pro-brain-type natriuretic peptide (NT-proBNP) and haemoglobin A1c levels.

\section{Echocardiographic data}

Trained sonographers performed transthoracic echocardiography to obtain cardiac images according to the recommendations of the American Society of Echocardiography and European Association of Cardiovascular Imaging (ASE/EACVI) guidelines. ${ }^{14} \mathrm{LV}$ systolic function was assessed by M-mode echocardiographic estimation of the LV ejection fraction (EF). We also extracted M-mode echocardiography data for LV end-systolic dimension, LV end-diastolic dimension, interventricular septum thickness and posterior ventricular wall thickness. LV mass indexed to body surface area was also estimated from the end-diastolic LV cavity dimension and wall thickness which were determined by M-mode echocardiography.
LV diastolic function was assessed from the following parameters: mitral valve (MV) peak E-wave velocity, peak A-wave velocity, MV E/A ratio, MV deceleration time, pulsed-wave tissue Doppler imaging septal e' velocity, mitral E/e', left atrial (LA) dimension on M-mode echocardiographic parasternal short-axis view and right ventricular systolic pressure (RVSP). RVSP was calculated from the maximal velocity of the tricuspid regurgitation

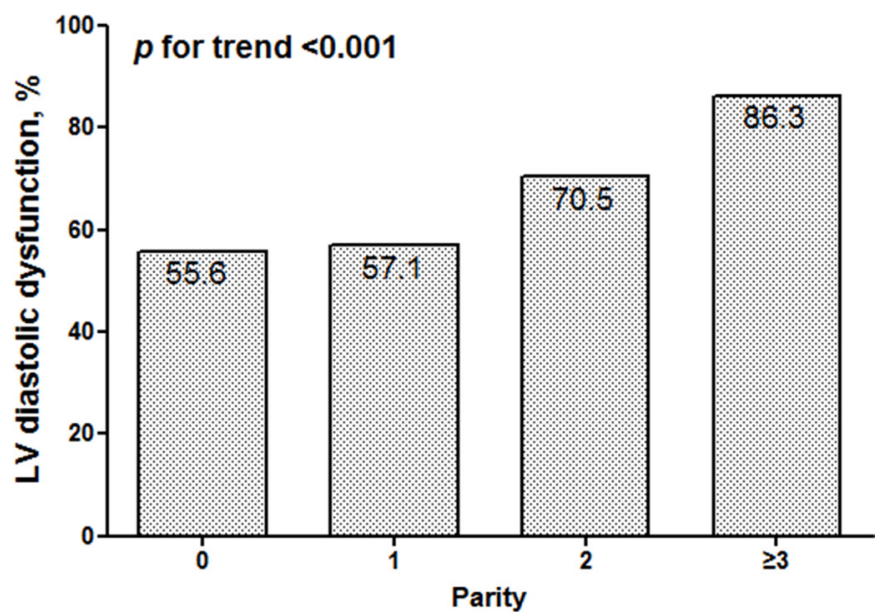

Figure 1 Prevalence of any-grade of diastolic dysfunction by levels of parity. The prevalence of left ventricular (LV) diastolic dysfunction was significantly increased according to parity number. 


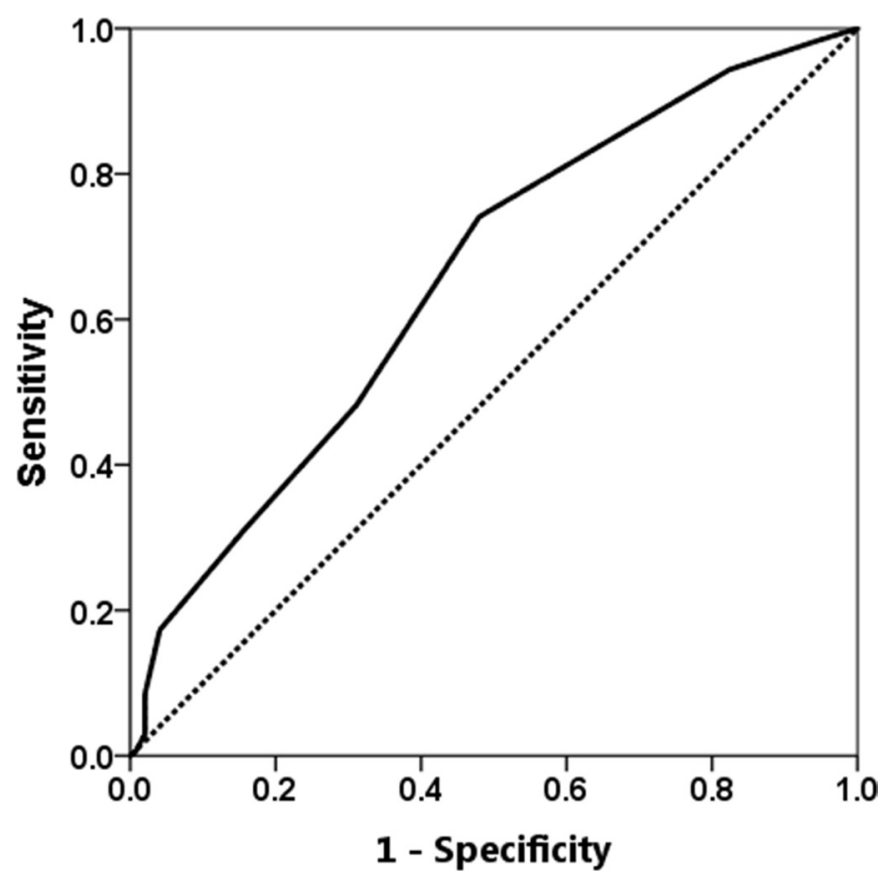

Figure 2 Receiver-operating curve (ROC) analysis. ROC analysis revealed that the best cut-off value of the parity number for predicting left ventricular diastolic dysfunction was 2.5 , with $74.1 \%$ sensitivity and $52.0 \%$ specificity (Area Under the Curve: 0.66 ; $95 \% \mathrm{Cl} 0.607$ to 0.706 ; $p<0.001$ ).

jet and the estimated right atrial pressure. LV diastolic dysfunction and the grading of $\mathrm{LV}$ diastolic function were evaluated according to the algorithms of the ASE/EACVI guidelines.

\section{Statistical analysis}

All categorical data were expressed as frequencies and percentages, whereas statistics for continuous variables were presented as means and SD. The Pearson $\chi^{2}$ test was used to compare categorical variables. The Student's $t$-test was used to compare continuous variables with normality, and the Mann-Whitney U-test was used to compare continuous variables without normality. The linear-by-linear association test was used to identify trends in the prevalence of LV diastolic dysfunction in association with parity number. We used receiver operating characteristic (ROC) curve analysis to determine the optimal cut-off parity number for predicting LV diastolic dysfunction. In addition, univariate analysis and subsequent multivariate logistic regression analysis were performed to evaluate the risk of LV diastolic dysfunction by adjusting for individual risk factors. Variables that were identified by univariate analysis as significant $(p<0.05)$ were included in the regression model. A p value less than 0.05 was considered statistically significant. Analysis was performed using SPSS V.21.0 (IBM).

\section{Patient and public involvement}

This study was conducted without patient and public involvement. The patients were not invited to comment on the study design and were not consulted to develop outcomes or interpret the results. Patients were not invited to contribute to the writing or editing of this document for readability or accuracy.

\section{RESULTS}

\section{Baseline characteristics}

The study included 960 women with suspected coronary artery disease. Their mean age was $63.5 \pm 11.4$ years, and mean parity number was $3.6 \pm 1.8$. There were 302 patients allocated to the low-parity group (parity 0 to $<3$ ) and 658 patients allocated to the multiparity group (parity $\geq 3$ ). The patients' baseline characteristics are presented in table 1 . Multiparity patients were significantly older than low-parity patients (66.6 \pm 9.9 vs $56.6 \pm 11.5$ year, respectively; $\mathrm{p}<0.001)$. Waist circumference was significantly higher in the multiparity than in the low-parity group. The proportions of multiparity patients with diabetes mellitus and hypertension were larger than the proportions of low-parity patients. The proportion of multiparity patients with coronary artery disease was also higher than that of the low-parity. The mean haemoglobin values

Table 3 Univariate and multivariate analyses of multiparity for predicting diastolic dysfunction

\begin{tabular}{|c|c|c|c|c|c|c|}
\hline & \multicolumn{3}{|c|}{ Univariate analysis } & \multicolumn{3}{|c|}{ Multivariate analysis } \\
\hline & OR & $95 \% \mathrm{Cl}$ & $P$ value & OR & $95 \% \mathrm{Cl}$ & $P$ value \\
\hline $\begin{array}{l}\text { Multiparity } \\
(n \geq 3)\end{array}$ & 3.10 & 2.141 to 4.491 & $<0.001$ & 1.80 & 1.053 to 3.081 & 0.032 \\
\hline Age (>60year) & 14.32 & 8.805 to 23.283 & $<0.001$ & 9.11 & 4.732 to 17.528 & $<0.001$ \\
\hline $\mathrm{BMI} \geq 25 \mathrm{~kg} / \mathrm{m}^{2}$ & 1.70 & 1.157 to 2.510 & 0.007 & 1.80 & 1.055 to 3.080 & 0.031 \\
\hline Diabetes mellitus & 9.24 & 3.994 to 21.373 & $<0.001$ & 6.24 & 1.409 to 27.598 & 0.016 \\
\hline $\begin{array}{l}\text { Coronary artery } \\
\text { disease }\end{array}$ & 4.50 & 2.994 to 6.762 & $<0.001$ & 1.84 & 1.050 to 3.081 & 0.039 \\
\hline WCC $>10 \times 10^{9} / \mathrm{L}$ & 2.86 & 1.452 to 5.636 & 0.002 & 0.885 & 0.344 to 2.278 & 0.799 \\
\hline
\end{tabular}

BMI, body mass index; WCC, white cell count. 
were significantly lower, and the NT-proBNP values were significantly higher in the multiparity patients than in the low-parity patients. The differences between values for blood pressure, heart rate, bilateral ABIs, incidence of atrial fibrillation and other laboratory findings were not significant.

\section{Parity difference in LV diastolic dysfunction}

Table 2 shows the echocardiographic characteristics of the multiparity and the low-parity patients. The proportion of multiparity patients with $\mathrm{LV}$ diastolic dysfunction was higher than that of the low-parity patients $(86.3 \%$ vs $67.0 \%$, respectively; $\mathrm{p}<0.001)$. The trend to gradual increase in LV diastolic dysfunction according to parity number was significant (figure $1, p$ for trend $<0.001$ ). The parameters representative for LV diastolic dysfunction showed significantly worse values for the multiparity patients than the parameters of the low-parity patients as follows: the multiparity patients showed a significantly lower MV E velocity, E/A ratio and septal $\mathrm{e}^{\prime}$ velocity and significantly higher $\mathrm{E} / \mathrm{e}^{\prime}$ ratio and RVSP than the low-parity patients. In addition, LV dimensions in both end-systole and end-diastole were significantly larger in the multiparity than in the low-parity patients. Multiparity patients showed a significantly lower LV EF value and a significantly higher LV mass index value than the low-parity patients.

\section{Multiparity as a predictor of LV diastolic dysfunction}

ROC curve analysis identified a parity of 2.5 as the cut-off value for predicting $\mathrm{LV}$ diastolic dysfunction in multiparity patients (figure 2).

Univariate analysis (table 3) identified the following parameters to be associated with $\mathrm{LV}$ diastolic dysfunction: multiparity (OR $3.10,95 \%$ CI 2.141 to $4.491, \mathrm{p}<0.001$ ), age older than 60 years, BMI $\geq 25 \mathrm{~g} / \mathrm{m}^{2}$ and heart rate; and comorbidities including hypertension, diabetes mellitus and coronary artery disease. White cell count was also associated with LV diastolic dysfunction.

By multivariate logistic regression analysis (table 3), multiparity was found to show a 1.80 -fold increased risk for LV diastolic dysfunction after adjusting for other relevant factors (OR 1.80, 95\% CI 1.053 to 3.081, $\mathrm{p}=0.032$ ). Older age ( $>60$ year) was found to show a 9.1-fold increased risk of LV diastolic dysfunction. Hypertension and diabetes mellitus were also significant predictors of LV diastolic dysfunction. Coronary artery disease increased the risk of LV diastolic dysfunction independently by 1.84 -fold.

We also performed univariate analysis and multivariate logistic regression analysis for 1:1 age-matched low-parity $(\mathrm{n}=302)$ and multiparity $(\mathrm{n}=302)$ patients. Online supplementary table 1 demonstrates that after adjusting for relevant factors, multiparity and coronary artery disease were independent predictors of increased risk for LV diastolic dysfunction (OR 1.74, 95\% CI 1.059 to 2.845, $\mathrm{p}=0.029$; OR $2.15,95 \%$ CI 1.280 to $3.614, \mathrm{p}=0.004$, respectively).

\section{DISCUSSION}

Consistent with the knowledge that repeated pregnancies are significantly associated with cardiovascular disease in women, ${ }^{78}$ the prevalence of LV diastolic dysfunction was higher in the multiparity than in the low-parity patients in our study. LV diastolic dysfunction gradually increased with parity number, and a parity of 2.5 was the best cut-off value for predicting development of $\mathrm{LV}$ diastolic dysfunction. Moreover, multiparity $(\mathrm{n} \geq 3)$ was a good predictor for detecting $\mathrm{LV}$ diastolic dysfunction. Advanced age (>60year), hypertension, diabetes mellitus, BMI $\geq 25$, heart rate and coronary artery disease were also independent predictors of LV diastolic dysfunction. After additional analysis of the age-matched groups, multiparity remained an independent predictor of LV diastolic dysfunction.

\section{LV diastolic dysfunction and multiparity}

The mechanisms underlying the effect of multiparity on LV diastolic dysfunction are not well understood and are controversial. During pregnancy, the reninangiotensin-aldosterone system undergoes significant changes. ${ }^{15}$ The upregulation of renin first occurs; and oestrogen, a steroid hormone essential for maintaining pregnancy, is produced by the placenta. Oestrogen leads to increased synthesis of angiotensinogen which leads to an increased serum angiotensin-II level. The activation of the renin-angiotensin-aldosterone system during pregnancy leads to increased retention of water and sodium in the mother. These hormonal changes can lead to changes in the cardiovascular system to adapt to an increasing blood supply, thus enabling maintenance of the pregnancy. However, the long-term cardiovascular effects resulting from these adaptations to repeated pregnancies have remained unclear. There have been several studies showing long-term changes in maternal cardiovascular system after pregnancy, and Keskin et $a l$ have demonstrated that grand multiparity over four in Turkish population is an independent risk factor for LV diastolic dysfunction. ${ }^{16}$ Lv et al suggested that the parity number is inversely associated with cardiovascular mortality until the parity number reaches four births. ${ }^{17}$ After four births, the linear relationship appeared to rebound. On the contrary, Catov et al demonstrated that parous women had higher risk of cardiovascular disease than nulliparous women, and parity number five or more birth had highest cardiovascular prevalence of cardiovascular disease. ${ }^{18}$ The outcome of our study was prevalence of LV diastolic dysfunction only, and may differ from the results of cardiovascular disease or cardiovascular mortality in those previous studies. Our findings show that the prevalence of LV diastolic dysfunction increased according to parity number. The possible mechanism of these findings by which repeated pregnancies effect LV diastolic dysfunction may be related to changes in various hormones. Retention of water and sodium according to the activation of the renin-angiotensin-aldosterone system and the adaptive changes in association with each 
pregnancy might lead to LV diastolic impairment. In addition, oestrogen has been known to attenuate myocardial hypertrophy, ${ }^{19}$ and myocardial hypertrophy according to change of oestrogen might be related to LV diastolic dysfunction in women with repeated pregnancies. Actually, our study demonstrated that more multiparous patients than low-parity patients had LV hypertrophy, based on the increased LV mass index value. Relaxin, a hormone produced during pregnancy, also contributes to the physiological cardiovascular adaptations, because of its vasodilating, angiogenic and antifibrotic properties, the last of which protects the left ventricle from fibrosis. ${ }^{20}$ All these hormonal upregulations during repeated pregnancies might be related to the later development of irreversible LV diastolic dysfunction.

\section{Other related factors predisposing to LV diastolic dysfunction}

The prevalence of LV diastolic dysfunction is known to increase with age. ${ }^{21}$ Our findings demonstrated that women aged older than 60 years had a significantly increased risk of LV diastolic dysfunction. In our study, the older the age, the more multiparity. Since LV diastolic dysfunction is exacerbated by the ageing process, we performed an age-matched 1:1 analysis for the low-parity and multiparity groups. After adjustment for age, multiparity remained an independent predictor for $\mathrm{LV}$ diastolic dysfunction. Age is an important risk factor for LV diastolic dysfunction, but our age-matched analysis allows us to emphasise that increased parity number is a risk for LV diastolic dysfunction. Our study also showed that diabetes mellitus increased the risk of LV diastolic dysfunction by 6.24 -fold, hypertension by 2.79 -fold and BMI $\geq 25 \mathrm{~kg} / \mathrm{m}^{2}$ by 1.80 -fold, showing a significant association. These factors are components of metabolic syndrome which is a known risk factor for cardiovascular disease. Moreover, metabolic syndrome has also been also shown in previous studies to be significantly associated with LV diastolic dysfunction and an independent risk factor of LV diastolic dysfunction ${ }^{122-24}$ which is consistent with our results.

Previous studies demonstrated that multiparity increased the risk of obstructive coronary artery disease in women, ${ }^{825} 26$ and coronary artery disease has been found to be associated with LV diastolic dysfunction. ${ }^{27}$ More multiparous than low-parity patients had obstructive coronary artery disease in our study, and coronary artery disease was independently associated with $\mathrm{LV}$ diastolic dysfunction by multivariate regression analysis. Since parity increases the risk of coronary artery disease, and coronary artery disease is an independent risk factor for LV diastolic dysfunction, multiparity is closely related to LV diastolic dysfunction.

This study has limitations that should be considered. First, this was a cross-sectional study which inherently cannot clarify the causal relationships between individual variables based on observations made at a single point in time. Second, based on the ASE/EACVI guidelines for the evaluation of LV diastolic function by echocardiography, the LA volume index is a key structural indicator of $\mathrm{LV}$ diastolic function. ${ }^{14}$ Because data on LA volumes were not available from the KoROSE registry, we used LA dimension data instead. Third, our findings might not be applicable to other populations because our study only studied patients with suspected coronary artery disease who had undergone coronary angiography. Finally, though socioeconomic status and lower education are associated with higher parity and which can also affect lifestyle behaviours and risk of cardiovascular disease, we could not analyse the differences of the socioeconomic status and education status between multiparity and low-parity groups because of insufficient data.

\section{CONCLUSIONS}

The prevalence of LV diastolic dysfunction was higher in multiparity than in low-parity women with suspected coronary artery disease. A parity of 2.5 was the cut-off value for predicting LV diastolic dysfunction. Moreover, multiparity (three or more pregnancies) was an independent risk factor for LV diastolic dysfunction. Therefore, the obstetric history of female adult patients is important, and LV diastolic dysfunction should be evaluated in multiparous women to assess the risk of cardiovascular disease and facilitate the initiation of appropriate treatment.

\section{Author affiliations}

${ }^{1}$ Chungbuk Regional Cardiovascular Center, Chungbuk National University Hospital, Cheongju, Chungcheongbuk-do, Korea

${ }^{2}$ Cardiovascular Center, Seoul National University Boramae Medical Hospital, Seoul, Korea

${ }^{3}$ Cardiovascular Center, Korea University Anam Hospital, Seoul, Korea ${ }^{4}$ Department of Cardiology, Chonnam National University Hospital, Gwangju, Korea ${ }^{5}$ Department of Cardiology, Gachon Medical School Gil Medical Center, Incheon, Korea

${ }^{6}$ Department of Cardiology, Hanllym University Chuncheon Sacred Heart Hospital, Chuncheon, Korea

${ }^{7}$ Department of Cardiology, Ewha Womans University Hospital, Seoul, Korea ${ }^{8}$ Department of Cardiology, Korea University Guro Hospital, Seoul, Korea

${ }^{9}$ Department of Cardiology, Chungnam National University Hospital, Daejeon, Korea

Contributors H-JK: conceptualisation, methodology, software, writing-original draft preparation. M-AK: visualisation, investigation, software, writing-reviewing and editing, supervision. H-LK: data curation, software, writing-reviewing and editing. WJS: investigation, data curation. MK: validation, data curation, software. S-MP, HJY, M-SS, K-SH, GJS, Y-HK, JON and J-OJ: validation, data curation.

Funding The authors have not declared a specific grant for this research from any funding agency in the public, commercial or not-for-profit sectors.

Competing interests None declared.

Patient consent for publication Obtained.

Ethics approval Institutional review board of each centre including Seoul National University Boramae Hospital.

Provenance and peer review Not commissioned; externally peer reviewed.

Data sharing statement № additional data are available.

Open access This is an open access article distributed in accordance with the Creative Commons Attribution Non Commercial (CC BY-NC 4.0) license, which permits others to distribute, remix, adapt, build upon this work non-commercially, and license their derivative works on different terms, provided the original work is properly cited, appropriate credit is given, any changes made indicated, and the use is non-commercial. See: http://creativecommons.org/licenses/by-nc/4.0/. 


\section{REFERENCES}

1. Kuznetsova T, Thijs L, Knez J, et al. Prognostic value of left ventricular diastolic dysfunction in a general population. J Am Heart Assoc 2014;3:e000789.

2. Kane GC, Karon BL, Mahoney DW, et al. Progression of left ventricular diastolic dysfunction and risk of heart failure. JAMA 2011;306:856-63.

3. Mottram PM, Marwick TH. Assessment of diastolic function: what the general cardiologist needs to know. Heart 2005;91:681-95.

4. Clapp JF, Seaward BL, Sleamaker RH, et al. Maternal physiologic adaptations to early human pregnancy. Am J Obstet Gynecol 1988;159:1456-60.

5. Mesa A, Jessurun $C$, Hernandez $A$, et al. Left ventricular diastolic function in normal human pregnancy. Circulation 1999;99:511-7.

6. Moran AM, Colan SD, Mauer MB, et al. Adaptive mechanisms of left ventricular diastolic function to the physiologic load of pregnancy. Clin Cardiol 2002;25:124-31.

7. Peters SAE, Yang L, Guo Y, et al. Pregnancy, pregnancy loss, and the risk of cardiovascular disease in Chinese women: findings from the China Kadoorie Biobank. BMC Med 2017;15:148.

8. Ness RB, Harris T, Cobb J, et al. Number of pregnancies and the subsequent risk of cardiovascular disease. $N$ Engl J Med 1993;328:1528-33.

9. Lewis CE, Funkhouser E, Raczynski JM, et al. Adverse effect of pregnancy on high density lipoprotein (HDL) cholesterol in young adult women. The CARDIA Study. coronary artery risk development in young adults. Am J Epidemiol 1996;144:247-54.

10. Brizzi P, Tonolo G, Esposito F, et al. Lipoprotein metabolism during normal pregnancy. Am J Obstet Gynecol 1999;181:430-4.

11. Desoye G, Schweditsch MO, Pfeiffer KP, et al. Correlation of hormones with lipid and lipoprotein levels during normal pregnancy and postpartum. J Clin Endocrinol Metab 1987;64:704-12.

12. Kim HL, Kim MA, Oh S, et al. Sex difference in the association between metabolic syndrome and left ventricular diastolic dysfunction. Metab Syndr Relat Disord 2016;14:507-12.

13. Rumberger JA. Coronary artery disease: a continuum, not a threshold. Mayo Clin Proc 2017;92:323-6.

14. Nagueh SF, Smiseth OA, Appleton CP, et al. Recommendations for the evaluation of left ventricular diastolic function by echocardiography: an update from the American Society of echocardiography and the european association of cardiovascular imaging. Eur Heart J Cardiovasc Imaging 2016;17:1321-60.
15. Irani RA, Xia Y. Renin angiotensin signaling in normal pregnancy and preeclampsia. Semin Nephrol 2011;31:47-58.

16. Keskin M, Avșar Ş, Hayıroğlu Mİ, et al. Relation of the number of parity to left ventricular diastolic function in pregnancy. Am J Cardiol 2017;120:154-9.

17. Lv H, Wu H, Yin J, et al. Parity and cardiovascular disease mortality: a dose-response meta-analysis of cohort studies. Sci Rep 2015;5:13411.

18. Catov JM, Newman AB, Sutton-Tyrrell K, et al. Parity and cardiovascular disease risk among older women: how do pregnancy complications mediate the association? Ann Epidemiol 2008;18:873-9.

19. Kilić A, Javadov S, Karmazyn M. Estrogen exerts concentrationdependent pro-and anti-hypertrophic effects on adult cultured ventricular myocytes. Role of NHE-1 in estrogen-induced hypertrophy. J Mol Cell Cardiol 2009;46:360-9.

20. Teichman SL, Unemori E, Teerlink JR, et al. Relaxin: review of biology and potential role in treating heart failure. Curr Heart Fail Rep 2010;7:75-82.

21. Fischer M, Baessler A, Hense HW, et al. Prevalence of left ventricular diastolic dysfunction in the community. Results from a Doppler echocardiographic-based survey of a population sample. Eur Heart $J$ 2003;24:320-8.

22. de las Fuentes L, Brown AL, Mathews SJ, et al. Metabolic syndrome is associated with abnormal left ventricular diastolic function independent of left ventricular mass. Eur Heart J 2007;28:553-9.

23. From AM, Scott CG, Chen $\mathrm{HH}$. Changes in diastolic dysfunction in diabetes mellitus over time. Am J Cardiol 2009;103:1463-6.

24. From AM, Scott CG, Chen HH. The development of heart failure in patients with diabetes mellitus and pre-clinical diastolic dysfunction a population-based study. J Am Coll Cardiol 2010;55:300-5.

25. Kim H-L, Kim M-A, Shim W-J, et al. Reproductive factors predicting angiographic obstructive coronary artery disease: the KoRean wOmen'S Chest Pain rEgistry (KoROSE). J Womens Health 2016;25:443-8.

26. Parikh NI, Cnattingius S, Dickman PW, et al. Parity and risk of laterlife maternal cardiovascular disease. Am Heart J 2010;159:215-21.

27. Maragiannis D, Schutt RC, Gramze NL, et al. Association of left ventricular diastolic dysfunction with subclinical coronary atherosclerotic disease burden using coronary artery calcium scoring. J Atheroscler Thromb 2015;22:1278-86. 